A. Noda

Nagoya Math. J.

Vol. 59 (1975), 65-76

\title{
GAUSSIAN RANDOM FIELDS WITH PROJECTIVE INVARIANCE
}

\author{
AKIO NODA
}

\section{§ 1. Introduction}

We shall consider the class of Gaussian random fields $\boldsymbol{X}_{\alpha}=\{X(A)$; $\left.A \in \boldsymbol{R}^{n}\right\}$ such that $E\{X(A)-X(B)\}=0$ and $E\left\{(X(A)-X(B))^{2}\right\}=|A-B|^{\alpha}$ $(0<\alpha<2)$, where $|A-B|$ denotes the Euclidean distance between two points $A$ and $B$.

The correlation function of $X_{\alpha}$ under the condition $X(P)=0$, which is denoted by $\rho_{\alpha}(A, B \mid P)$, is

$$
\rho_{\alpha}(A, B \mid P)=\left(|A-P|^{\alpha}+|B-P|^{\alpha}-|A-B|^{\alpha}\right) / 2|A-P|^{\alpha / 2}|B-P|^{\alpha / 2} .
$$

Clearly, the equality

$$
\text { (*) } \quad \rho_{\alpha}(T A, T B \mid T P)=\rho_{\alpha}(A, B \mid P)
$$

holds for a translation or an orthogonal transformation $T$ on $\boldsymbol{R}^{n}$. This property corresponds to stationarity and isotropy of $\boldsymbol{X}_{\alpha}$ (see [10]). Moreover, we have (*) when $T$ is a similar transformation or an inversion with center $P$. We shall call these properties projective invariance.

In $\S 2$, we shall show that projective invariance characterizes the class of $\boldsymbol{X}_{\alpha}$ 's (Theorem 1) and that only the above mentioned transformations satisfy ( $*$ ) unless $\alpha=n=1$ (Theorem 2).

In $\S 3$, we shall apply projective invariance to the prediction problem and get the new formulas. In particular, the two important properties of $\boldsymbol{X}_{\alpha}$-non-deterministic property and Markov property-will be mentioned and be used in $\S 4$.

In $\S 4$, we shall consider the conditional correlation function of $X_{\alpha}$. For a closed set $e$ in $R^{n}$, we calculate the conditional expectation of $X(A)$ relative to the $\sigma$-field generated by $\{X(P) ; P \in e\}$, which is denoted by $\mu(A \mid e)$. Then the conditional correlation function relative to $e$ is defined as follows:

Received June 14, 1974. 


$$
\rho_{\alpha}(A, B \mid e)=\frac{E\{(X(A)-\mu(A \mid e))(X(B)-\mu(B \mid e))\}}{\left[E\left\{(X(A)-\mu(A \mid e))^{2}\right\} E\left\{(X(B)-\mu(B \mid e))^{2}\right\}\right]^{1 / 2}} .
$$

We are interested in the problem to determine the transformation $T$ on $\boldsymbol{R}^{n}$ satisfying the following equality:

$$
\rho_{\alpha}(T A, T B \mid T e)=\rho_{\alpha}(A, B \mid e) .
$$

It is easy to see that translations, orthogonal transformations, similar transformations and inversions with the center in $e$ satisfy $(* *)$ (Theorem 3). We are able to determine $T$ satisfying ( $* *$ ) in the case $\mathrm{n}=1$ (Theorem 4). There occurs a remarkable difference between the cases $\alpha=1$ and $\alpha \neq 1$, and this is due to Markov property. The case $\alpha=1$ in Theorem 4 corresponds to the principle of projective invariance of the Brownian motion ([5]) which was the starting point of our study.

\section{\$2. Gaussian random field $X_{\alpha}$ with projective invariance}

Let $X=\left\{X(A) ; A \in \boldsymbol{R}^{n}\right\}$ be a Gaussian random field such that $X(A)$ $-X(B)$ has mean 0 and variance $r(A, B)$, where $r(A, B)$ is a function on $\boldsymbol{R}^{n} \times \boldsymbol{R}^{n}$ satisfying the following conditions:

$$
\left\{\begin{array}{l}
r(A, B)=r(B, A), r(A, A)=0, r(A, B) \geq 0, \text { and } r \text { is a function } \\
\text { of negative type, i.e. } \sum a_{i} a_{j} r\left(A_{i}, A_{j}\right) \leq 0 \text { for any } A_{i} \in R^{n} \text { and any } \\
a_{i} \in R^{1} \text { such that } \sum a_{i}=0(i=1,2, \cdots, N) .
\end{array}\right.
$$

We assume in the sequel that $r(A, B)$ is continuous and $r(A, B) \not \equiv 0$.

With the above assumptions we can speak of the probability law only for the system of the differences $\left\{X(A)-X(B) ; A, B \in \boldsymbol{R}^{n}\right\}$. Therefore, we need to impose an additional condition, say $X(P)=0$, in order to determine $X(A)$ uniquely. (In this section, by $P$ we denote an arbitrarily fixed point in $\boldsymbol{R}^{n}$.)

The covariance function of $\boldsymbol{X}$ is

$$
E[X(A) X(B) \mid X(P)=0]=(r(A, P)+r(B, P)-r(A, B)) / 2,
$$

and the correlation function of $\boldsymbol{X}$ is defined by

$$
\begin{aligned}
\rho(A, B \mid P) & \equiv E\left[\frac{X(A)}{r(A, P)^{1 / 2}} \cdot \frac{X(B)}{r(B, P)^{1 / 2}} \mid X(P)=0\right] \\
& =\frac{r(A, P)+r(B, P)-r(A, B)}{2 r(A, P)^{1 / 2} r(B, P)^{1 / 2}} .
\end{aligned}
$$


Definition 1. We say that a transformation $T$ on $\boldsymbol{R}^{n}$ leaves the correlation function of $\boldsymbol{X}$ invariant if the equality

$$
\rho(T A, T B \mid T P)=\rho(A, B \mid P)
$$

holds for any $A, B$ in $\boldsymbol{R}^{n}$. If all the translations, orthogonal transformations, similar transformations and inversions with center $P$ leave the correlation function of $\boldsymbol{X}$ invariant, we say $\boldsymbol{X}$ has projective invariance.

THEOREM 1. $\boldsymbol{X}$ has projective invariance if and only if

$$
r(A, B)=\mid \text { const. }|\cdot| A-\left.B\right|^{\alpha} \quad(0<\alpha \leq 2) .
$$

The proof of the "if" part is clear. The "only if" part is divided into the following two lemmas.

LEMMA 1. Both translations and orthogonal transformations leave the correlation function of $\boldsymbol{X}$ invariant if and only if $r(A, B)$ depends only on $|A-B|$, i.e. $r(A, B)=r(|A-B|)$.

The proof of Lemma 1 is elementary, so is omitted.

LEMMA 2. If all the translations, orthogonal transformations and similar transformations leave the correlation function of $\boldsymbol{X}$ invariant, then (4) holds.

Proof. By Lemma 1, $r(A, B)=r(|A-B|)$. We can easily show that $r(x)>0$ whenever $x>0$. Without loss of generality, we can assume that $r(1)=1$. Since similar transformations leave the correlation function invariant, we have $\rho(-b, a b \mid 0)=\rho(-1, a \mid 0)$ for any $a, b>0$. By (2), we have

$$
\begin{aligned}
\rho(-b, a b \mid 0) & =(r(b)+r(a b)-r((a+1) b)) / 2 r(b)^{1 / 2} r(a b)^{1 / 2}, \\
\rho(-1, a \mid 0) & =(1+r(a)-r(a+1)) / 2 r(a)^{1 / 2} .
\end{aligned}
$$

Hence,

$$
\begin{aligned}
& \left\{(r(a) r(b))^{1 / 2}-r(a b)^{1 / 2}\right\}\left\{r(b)^{1 / 2}-(r(a) r(a b))^{1 / 2}\right\} \\
& \quad=r(a)^{1 / 2} r((a+1) b)-(r(b) r(a b))^{1 / 2} r(a+1) .
\end{aligned}
$$

Therefore, $r(a b)=r(a) r(b)$ yields $r((a+1) b)=r(a+1) r(b)$. By induction, we see that $r(n b)=r(n) r(b)$ for any integer $n$. This implies that $r(q b)$ $=r(q) r(b)$ for any rational number $q$. It follows from the continuity of 
$r(x)$ that $r(a b)=r(a) r(b)$ for any $a, b>0$. Thus, $r(x)=x^{\alpha}$ and the fact that the condition (1) implies $0<\alpha \leq 2$ is well known.

(Q.E.D.)

The random field $X$ which corresponds to $r(A, B)=|A-B|^{\alpha}$ is denoted by $\boldsymbol{X}_{\alpha}$ and the associated correlation function is denoted by $\rho_{\alpha}$ as in $\S 1$. Since $\boldsymbol{X}_{2}$ is a trivial random field, we shall consider only the case $0<\alpha<2$ in the sequel.

We introduce a notation. $\mathscr{T}$ is the transformation group generated by translations, orthogonal transformations, similar transformations and inversions. $\left.T\right|_{D}$ is the restriction of a transformation $T$ to a subset $D$ of $\boldsymbol{R}^{n}$. For a transformation $T$ such that $T A=\tilde{T} A$ on $D$ for some $\tilde{T} \in \mathscr{T}$, we say $\left.T\right|_{D}$ belongs to $\left.\mathscr{T}\right|_{D}$.

THEOREM 2. If a transformation $T$ leaves the correlation function of $\boldsymbol{X}_{\alpha}$ invariant, then $T$ belongs to $\mathscr{T}$ unless $n=\alpha=1$. For $n=\alpha=1$, $T_{1(-\infty, P]}$ and $T_{1[P,+\infty)}$ belong to $\mathscr{T}_{1(-\infty, P]}$ and $\mathscr{T}_{\mathrm{I}[P,+\infty)}$, respectively.

For the proof of Theorem 2, we use the following properties of $\rho_{\alpha}$. On $R^{2}$, fix $B=(1,0)$ and $P=(0,0)$ as the origin, and consider the variable $A=(r, \theta)$ with the polar coordinates. Then, we have

$$
\rho_{\alpha}(A, B \mid P)=\left(r^{\alpha / 2}+r^{-\alpha / 2}-\left(r+r^{-1}-2 \cos \theta\right)^{\alpha / 2}\right) / 2 .
$$

i) When $r$ is fixed, $\rho_{\alpha}$ is strictly increasing in $0 \leq \theta \leq \pi$.

ii) When $\theta(\leqq \pi / 2)$ is fixed, $\rho_{\alpha}$ is strictly increasing in $0 \leq r \leq 1$.

iii) When $\theta=\pi, \rho_{\alpha}$ is strictly decreasing, identically 0 and strictly increasing in $0 \leq r \leq 1$ according as $\alpha>1, \alpha=1$ and $\alpha<1$.

iv) If $\alpha>1, \rho_{\alpha}$ takes the minimal value $1-2^{\alpha-1}$ at $A=-B$. If $\alpha \leq 1$, the minimal value of $\rho_{\alpha}$ is 0 .

Proof of Theorem 2. For $T$ in Theorem 2, take the translation $T_{0}$ such that $T_{0}(T P)=P$. Then, $T_{0} \circ T$ leaves the correlation function invariant and fixes the point $P$. Therefore, we have only to prove Theorem 2 under the assumption $T P=P$.

The proof in the case $n=1$ is simple. We show here only the case $n=2$. For a general $n$ we can prove in a similar manner. On $\boldsymbol{R}^{2}$ we use the polar coordinates $(r, \theta)$ with origin $P$.

When $\alpha \neq 1$, we first claim that $\left.\left.T\right|_{S} \in \mathscr{T}\right|_{S}$ for a circle $S=\{r=a\}$. We may assume that $T(a, 0)=(a, 0)$. For $T$ which does not fix the point $(a, 0)$, we find a transformation $T_{1}$ in $\mathscr{T}$ such that $T_{1}(T(a, 0))=(a, 0)$ and 
$T_{1} P=P$, and consider $T_{1} \circ T$ instead of $T$. Under this assumption we show that $T(a, \pi)=(a, \pi)$. When $\alpha>1, \rho_{\alpha}(A,(a, 0) \mid P)\left(=\rho_{\alpha}(T A,(a, 0) \mid P)\right)$ takes the minimal value only at $(a, \pi)$, so we have $T(a, \pi)=(a, \pi)$. While, when $\alpha<1$, we describe the contour $\left\{A ; \rho_{\alpha}(A,(a, 0) \mid P)=\rho_{\alpha}((a, \pi),(a, 0) \mid P)\right\}$ and see that it crosses itself just at $(a, \pi)$. Hence we have $T(a, \pi)=(a, \pi)$ by the fact that $T$ is a homeomorphism and leaves the contour invariant. Since $(a, 0)$ and $(a, \pi)$ are invariant under $T$, we see that $T(a, \pi / 2)$ is either $(a, \pi / 2)$ or $(a,-\pi / 2)$ by using the properties (i), (ii) of $\rho_{\alpha}$. First assume that $T(a, \pi / 2)=(a, \pi / 2)$. Since $(a, 0)$ and $(a, \pi / 2)$ are invariant under $T$, so is $(a, \pi / 4)$. Thus all the points $\left(a, k \pi 2^{-n}\right)$ are invariant under $T\left(-2^{n}<k \leq 2^{n} ; n=0,1,2, \cdots\right)$. By the continuity of $T$, we have $T(a, \theta)=(a, \theta)$ for any point $(a, \theta)$ in $S$. On the other hand, if $T(a, \pi / 2)$ $=(a,-\pi / 2)$, we can prove that $T(a, \theta)=(a,-\theta)$ by the similar argument. Namely, we have proved that $\left.\left.T\right|_{S} \in \mathscr{T}\right|_{s}$.

For any $r_{1} \neq r_{2}$, we can write $\left.T\right|_{\left\{r=r_{i}\right\}}\left(r_{i}, \theta\right)=\left(r_{i}^{\prime}, g_{i}(\theta)\right)$ with some $r_{i}^{\prime}$ $(i=1,2)$. It is easy to show that $g_{1}(\theta) \equiv g_{2}(\theta)$. Therefore, $T$ is expressed in the form $T(r, \theta)=(f(r), g(\theta))$. Clearly, $f(r)$ is either $c r$ or $c / r$, and $g(\theta)$ is either $\theta+\theta_{0}$ or $-\theta+\theta_{0}$ ( $c$ and $\theta_{0}$ are constants.). Thus we have proved that $T \in \mathscr{T}$ when $\alpha \neq 1$.

When $\alpha=1$, we see that $T_{1 \ell} \in \mathscr{T}_{1 \ell}$ for a half-line $\ell=\left\{\theta=\theta_{0}\right\}$ by using the fact that $\ell$ is the contour $\left\{A ; \rho_{1}\left(A,\left(1, \theta_{0}+\pi\right) \mid P\right)=0\right\}$. We can write $\left.T\right|_{\left\{\theta=\theta_{i}\right\}}\left(r, \theta_{i}\right)=\left(f_{i}(r), \theta_{i}^{\prime}\right)$, where we set $T\left\{\theta=\theta_{i}\right\}=\left\{\theta=\theta_{i}^{\prime}\right\} \quad(i=1,2)$. It is easy to show that $f_{1}(r)=f_{2}(r)$ unless $\left|\theta_{1}-\theta_{2}\right|=\pi$. Therefore, $T$ is expressed in the form $T(r, \theta)=(f(r), g(\theta))$, so $T$ must belong to $\mathscr{T}$.

(Q.E.D.)

\section{§3. Prediction formulas and Markov property of $\boldsymbol{X}_{\alpha}$}

For a closed set $e$ in $\boldsymbol{R}^{n}$, we set

$$
\begin{aligned}
\mu(A \mid e) & =E[X(A) \mid X(P) ; P \in e], \\
\sigma^{2}(A \mid e) & =E\left\{(X(A)-\mu(A \mid e))^{2}\right\} .
\end{aligned}
$$

Let a point $P_{0}$ be chosen arbitrarily in $e$. Under the additional condition $X\left(P_{0}\right)=0$, the conditional expectation $\mu(A \mid e)$ relative to the $\sigma$-field generated by $\{X(P) ; P \in e\}$ is calculated.

Let $T_{*}$ be the inversion with respect to the unit sphere with center $P_{0}$. The simple application of projective invariance gives us the following lemma. 
LEMMA 3. If the conditional expectation $\mu(A \mid e)$ is expressed in the form

$$
\mu(A \mid e)=\int_{e} X(P) d F_{A}(P)
$$

with a signed measure $d F_{A}(P)$ on $e$ depending on $A$, then we have

$$
\mu\left(T_{*} A \mid T_{*} e\right)=\int_{e} X\left(T_{*} P\right)\left|P-P_{0}\right|^{\alpha}\left|A-P_{0}\right|^{-\alpha} d F_{A}(P),
$$

and

$$
\sigma^{2}\left(T_{*} A \mid T_{*} e\right)=\left|A-P_{0}\right|^{-2 \alpha} \sigma^{2}(A \mid e) .
$$

By virtue of Lemma 3 we can obtain the new formulas for

$$
\mu\left(T_{*} A \mid T_{*} e\right) \text { and } \sigma^{2}\left(T_{*} A \mid T_{*} e\right)
$$

from the known formulas for $\mu(A \mid e)$ and $\sigma^{2}(A \mid e)$. For example, when $e$ is a spherical surface $S$ and $A$ is its center, $T_{*} S$ becomes a hyperplane. So the simple formula

$$
\mu(A \mid S)=\int_{S} X(P) d \mu(P)
$$

( $d \mu$ is the uniform probability measure on $S$.)

gives us the prediction formulas relative to a hyperplane $L$.

Put $A=(1,0, \cdots, 0), L=\left\{\left(x_{1}, \cdots, x_{n}\right) ; x_{1}=0\right\}$ and $P=\left(0, x_{2}, \cdots, x_{n}\right)$. Then,

$$
\begin{aligned}
\mu(A \mid L)= & \pi^{-(n-1) / 2} \Gamma\left(n-1+\frac{\alpha}{2}\right) \Gamma\left(\frac{n-1+\alpha}{2}\right)^{-1} \\
& \times \int_{L} X(P)|P-A|^{-2 n+2-\alpha} d x_{2} \cdots d x_{n}, \\
\sigma^{2}(A \mid L)= & \Gamma\left(n-1+\frac{\alpha}{2}\right) \Gamma\left(\frac{n-1}{2}\right) / 2 \Gamma\left(\frac{n-1+\alpha}{2}\right) \Gamma(n-1) .
\end{aligned}
$$

When $e$ is a half-space $H$, M. I. Fortus ([2]) gave the formula:

$$
\sigma^{2}(A \mid H)=2^{\alpha-1} \Gamma\left(\frac{n}{2}\right)|A-H|^{\alpha} / \Gamma\left(\frac{2-\alpha}{2}\right) \Gamma\left(\frac{n+\alpha}{2}\right) .
$$

Applying Lemma 3 to (5), we have

$$
\begin{array}{r}
\sigma^{2}\left(A \mid \mathscr{S}^{c}\right)=\Gamma\left(\frac{n}{2}\right) R^{-\alpha}\left(R^{2}-|A-O|^{2}\right)^{\alpha} / 2 \Gamma\left(\frac{2-\alpha}{2}\right) \Gamma\left(\frac{n+\alpha}{2}\right)>0 \\
\quad \text { for } A \in \mathscr{S},
\end{array}
$$


where $\mathscr{S}$ is the sphere $\{P ;|P-O|<R\}$. This implies the following proposition.

Proposition 1. $\boldsymbol{X}_{\alpha}$ is non-deterministic. That is, $\sigma(A \mid e)>0$ for any $e$ and any $A \notin e$.

We cite here the following two formulas from [2], [4] and [9] which will be used in the next section.

Let $H$ be the half-space $\left\{\left(x_{1}, \cdots, x_{n}\right) ; x_{1} \leq 0\right\}$ and $A=(1,0, \cdots, 0)$. For $\alpha \neq 1$ or for an even $n$,

$$
\mu(A \mid H)=\pi^{-(n / 2)-1} \Gamma\left(\frac{n}{2}\right) \sin \left(\frac{\alpha+n}{2} \pi\right)
$$

$$
\times \int_{0}^{\infty} p^{-(\alpha+n) / 2} d p\left\{N(p)-\sum_{j=0}^{[(n-2+\alpha) / 2]} \frac{p^{j}}{j !} N^{(j)}(0)\right\},
$$

where

$$
N(p)=\int_{R^{n-1}} X((-p, y))\left((p+1)^{2}+|y|^{2}\right)^{-n / 2} d y,
$$

and $N^{(j)}$ is the $j$-th derivative of $N(p)$.

Next, let $\mathscr{S}$ be the sphere $\{P ;|P-O|<R\}$ and $A$ be any point of $\mathscr{S}$. If $\alpha=1$ and $n$ is odd, we have

$$
\begin{aligned}
\mu\left(A \mid \mathscr{S}^{c}\right)= & \frac{\left(R^{2}-|A-O|^{2}\right)^{(n+1) / 2}}{((n-1) / 2) !} \\
& \times\left.\left(-\frac{1}{2 r} \frac{d}{d r}\right)^{(n-1) / 2}\left(\int_{S(r)} \frac{r^{n-2} X(P)}{|A-P|^{n}} d \mu(P)\right)\right|_{r=R},
\end{aligned}
$$

where $S(r)=\{P ;|P-O|=r\}(r>R)$ and $d \mu$ is the uniform probability measure on $S(r)$.

From these formulas we can see the following important property of $X_{\alpha}$.

Proposition 2. $\boldsymbol{X}_{\alpha}$ has Markov property if and only if $\alpha=1$ and $n$ is odd.

This proposition has been proved in [6] and [7]. Furthermore we may expect that $\boldsymbol{X}_{\alpha}$ has anti-Markov property for $\alpha \neq 1$ or for an even $n$. For an open set $U$ in $\boldsymbol{R}^{n}$ we define a sub- $\sigma$-field $\boldsymbol{F}(U)$ by

$$
\begin{aligned}
F(U)= & \text { the smallest } \sigma \text {-field for which all the } X(A)(A \in U) \text { is } \\
& \text { measurable. }
\end{aligned}
$$


For each domain $D$ in $\boldsymbol{R}^{n}$, we introduce the following four sub- $\sigma$-fields in order to explain anti-Markov property:

$$
\begin{aligned}
F^{-}(D) & =\bigcap_{\varepsilon>0} F\left(D_{\varepsilon}\right) \text {, where } D_{\varepsilon} \text { means an } \varepsilon \text {-neighborhood of } D . \\
F^{+}(D) & =\bigcap_{\varepsilon>0} F\left(\left(D^{C}\right)_{\varepsilon}\right) . \\
\partial F(D) & =\bigcap_{\varepsilon>0} F\left((\partial D)_{\varepsilon}\right), \text { where } \partial D \text { means the boundary of } D . \\
F^{+/-}(D) & =\text { the smallest splitting field of } \boldsymbol{F}^{+}(D) \text { upon } \boldsymbol{F}^{-}(D) \text { (see [6]). }
\end{aligned}
$$

Following to Dym-McKean [1], we call the case $\boldsymbol{F}^{+/-}(D)=\boldsymbol{F}^{-}(D)$ antiMarkovian, while the case $\boldsymbol{F}^{+/-}(D)=\partial \boldsymbol{F}(D)$ is termed Markovian. For an even $n$, it is known that $X_{1}$ has anti-Markov property ([6]). For $n=1$ and $\alpha \neq 1, X_{\alpha}$ has anti-Markov property ${ }^{\circ}$ We can prove this fact by the use of the canonical representation of $\boldsymbol{X}_{\alpha}$ due to Molchan-Golosov [8], and we shall use it in the proof of Theorem 4 .

\section{§4. Invariance for the conditional correlation function of $\boldsymbol{X}_{\alpha}$}

For a closed set $e$ in $\boldsymbol{R}^{n}$, we set

$$
X(A)=\mu(A \mid e)+\sigma(A \mid e) \xi(A \mid e) .
$$

The conditional correlation function of $\boldsymbol{X}_{\alpha}$ relative to $e$ has been defined in $\S 1$ by

$$
\rho_{\alpha}(A, B \mid e)=E\{\xi(A \mid e) \xi(B \mid e)\} \text {. }
$$

We can easily show the following facts 1 ) $\sim 4$ ). In particular, 2) and 3) follow from the non-deterministic property.

1) $\rho_{\alpha}(A, B \mid e)=0$ for $A \in e$,

2) $\rho_{\alpha}(A, A \mid e)=1$ for $A \notin e$,

3) $\left|\rho_{\alpha}(A, B \mid e)\right|<1$ for $A \neq B$,

4) $\rho_{\alpha}(A, B \mid e)$ is continuous in $A$ and $B$ on $e^{C}$.

DeFinition 2. We say a transformation $T$ on $\boldsymbol{R}^{n}$ leaves the conditional correlation function of $\boldsymbol{X}_{\alpha}$ relative to $e$ invariant if the equality (**) holds for any $A, B \in \boldsymbol{R}^{n}$.

$$
\rho_{\alpha}(T A, T B \mid T e)=\rho_{\alpha}(A, B \mid e) .
$$

We note that for $T$ satisfying (**), a transformation $T^{\prime}$ such that $T^{\prime} e=T e$ and $\left.T^{\prime}\right|_{e c}=\left.T\right|_{e c}$ also satisfies the same equality (**).

THEOREM 3. For each e, $\mathscr{T}$ leaves the conditional correlation function 
of $\boldsymbol{X}_{\alpha}$ relative to $e$ invariant, provided that as to inversions we consider only the inversions with the center in $e$.

Theorem 3 is an easy consequence of Theorem 1 and the following simple lemma.

LEMma 4. If a transformation $T$ leaves the conditional correlation function relative to $e$ invariant, then for each $e^{\prime}$ containing $e T$ leaves the conditional correlation function relative to $e^{\prime}$ invariant.

LEMMA 5. For an open ball $\mathscr{S}$ in $\boldsymbol{R}^{n}$, if a transformation $T$ leaves the conditional correlation function relative to $\mathscr{S}^{C}$ invariant and if $T \mathscr{S}$ is also an open ball, then $\left.T\right|_{\mathscr{S}}$ belongs to $\left.\mathscr{T}\right|_{\mathscr{s}}$.

We note that in the case $n=1$ the condition that $T \mathscr{S}$ is an open ball is always fulfilled.

Proof of Lemma 5. Let $O$ be the center of $\mathscr{S}$ and $R$ be the radius. Assuming that $T O=O, T \mathscr{S}=\mathscr{S}$ and $T$ leaves the conditional correlation function relative to $\mathscr{S}^{c}$ invariant, we shall prove that $T$ is an orthogonal transformation on $\mathscr{S}$. Once this is established, we can prove Lemma 5 as follows. For $T$ in Lemma 5, we find $T_{0}$ in $\mathscr{T}$ such that $T_{0}(T O)=O$ and $T_{0}(T \mathscr{S})=\mathscr{S}$. Then $T_{0} \circ T$ satisfies the hypotheses of the above assertion, so $T_{0} \circ T$ is an orthogonal transformation on $\mathscr{S}$. Hence $\left.\left.T\right|_{\mathscr{S}} \in \mathscr{T}\right|_{\mathscr{s}}$.

For the proof of the above assertion, we calculate $\rho_{\alpha}\left(A, O \mid \mathscr{S}^{C}\right) \equiv$ $\tilde{\rho}_{\alpha}(|A-O|)$ explicitly by using the prediction formulas (5), (6) and (7) cited in $\S 3$. Then we can show that $(d / d a) \tilde{\rho}_{\alpha}(a)<0$ in $0<a<\varepsilon$, hence $\tilde{\rho}_{\alpha}$ is strictly decreasing in $(0, \varepsilon)$, where $\varepsilon$ is some sufficiently small positive number. Therefore, for any $A$ in some sufficiently small neighborhood of $O, \tilde{\rho}_{\alpha}(|T A-O|)=\tilde{\rho}_{\alpha}(|A-O|)$ yields $|T A-O|=|A-O|$.

We denote by $T_{(A)}$ the transformation in $\mathscr{T}$ such that $T_{(A)} A=O$, $T_{(A)} \mathscr{S}=\mathscr{S}$. The transformation $T_{(T A)} \circ T \circ T_{(A)}^{-1}$ has the same properties as $T$, so we have $\left|T_{(T A)}(T B)-O\right|=\left|T_{(A)} B-O\right|$ for small $\left|T_{(A)} B-O\right|$. On the other hand we can show that

$$
\begin{aligned}
\left|T_{(A)} B-O\right|= & R^{2}|A-B|\left\{|A-O|^{2}|B-O|^{2}\right. \\
& \left.+R^{4}-2|A-O||B-O| R^{2} \cos (\angle A O B)\right\}^{-1 / 2} .
\end{aligned}
$$

From the equalities:

$$
|T A-O|=|A-O|, \quad|T B-O|=|B-O|
$$


and

$$
\left|T_{(T A)}(T B)-O\right|=\left|T_{(A)} B-O\right|,
$$

we see that $\cos (\angle T A \cdot O \cdot T B)=\cos (\angle A O B)$. Hence $T$ is an orthogonal transformation on a sufficiently small neighborhood of $O$. Repeating this argument, it can be seen that $T$ is an orthogonal transformation on $\mathscr{S}$.

Let $n=1$. For a closed set $e$ in $R^{1}$, put $e^{C}=\cup_{i} I_{i}$, where $I_{i}$ 's are the connected components of the open set $e^{C}$.

THEOREM 4. If T leaves the conditional correlation function relative to $e$ invariant, then in the case $\alpha \neq 1,\left.T\right|_{e}$ belongs to $\left.\mathscr{T}\right|_{e}$. While, in the case $\alpha=1,\left.T\right|_{I_{i}}$ belongs to $\left.\mathscr{T}\right|_{I_{i}}$ for every $i$.

Proof. For each $i, T$ leaves the conditional correlation function relative to $I_{i}^{C}$ invariant by Lemma 4 . So we have $\left.\left.T\right|_{I_{i}} \in \mathscr{T}\right|_{I_{i}}$ by Lemma 5. In the case $\alpha=1$, the proof is completed. But in the case $\alpha \neq 1$, we must show that $\left.\left.T\right|_{e c} \in \mathscr{T}\right|_{e} c$. For any intervals $I_{i}$ and $I_{j}$, we shall show that if $\left.T\right|_{I_{i}}$ is the identical transformation, so is $\left.T\right|_{I_{j}}$. Then we see that $\left.\left.T\right|_{I_{i} \cup I_{j}} \in \mathscr{T}\right|_{I_{i} \cup I_{j}}$ by the fact that $\left.\left.T\right|_{I_{i}} \in \mathscr{T}\right|_{I_{i}}$. It follows from arbitrariness of $I_{i}$ and $I_{j}$ that $\left.\left.T\right|_{e^{o}} \in \mathscr{T}\right|_{e^{c}}$.

By considering $T_{0} \in \mathscr{T}$ such that $T_{0} I_{i}=(-1,0)$ and $T_{0} I_{j}=(a, \infty)$ $(a>0)$, we may prove the above assertion for $I_{i}=(-1,0)$ and $I_{j}=(a, \infty)$. Thus we assume that $T$ leaves the conditional correlation function relative to $((-1,0) \cup(a, \infty))^{C}$ invariant, that $\left.T\right|_{(-1,0)}$ is the identity, and that $\left.T\right|_{(a, \infty)}\left(\left.\in \mathscr{T}\right|_{(a, \infty)}\right)$ is not the identity. Then, we show that these assumptions contradict the anti-Markov property of $\boldsymbol{X}_{\alpha}$. Put $T(a, \infty)=(b, c)$. We show only the case $b>a$. All other cases are shown similarly.

Consider the transformation $T^{\prime}$ such that

$$
T^{\prime} x=\left.\frac{b}{a} T\right|_{(-a / b, 0) \cup(a,(a / b) c)}\left(\frac{p}{b} x\right) \quad \text { for } x \in(-1,0) \cup(b, c) .
$$

This transformation $T^{\prime}$ leaves the conditional correlation function relative to $(-\infty,-1] \cup[0, b] \cup[c, \infty)$ invariant, $T^{\prime}$ belongs to $\mathscr{T}$ on $(b, c)$ and $T^{\prime}$ is the identity on $(-1,0)$. Put $T^{\prime}(b, c)=\left(b_{1}, c_{1}\right)$. Then we see that $b_{1} / b$ $\geq b / a$. Next consider $T^{\prime \prime}$ such that $T^{\prime \prime}$ leaves the conditional correlation function relative to $(-\infty,-1] \cup\left[0, b_{1}\right] \cup\left[c_{1}, \infty\right)$ invariant, $T^{\prime \prime}$ belongs to $\mathscr{T}$ on $\left(b_{1}, c_{1}\right)$ and $T^{\prime \prime}$ is the identity on $(-1,0)$. Then we see that $b_{2} / b_{1}$ 
$\geq b / a$ if we put $T^{\prime \prime}\left(b_{1}, c_{1}\right)=\left(b_{2}, c_{2}\right)$. Repeating this procedure, we have the following equalities: for any $A \in(-1,0)$ and any $B \in(a, \infty)$,

$$
\begin{aligned}
\rho_{\alpha}(A, & B \mid(-\infty,-1] \cup[0, a])=\rho_{\alpha}(A, T B \mid(-\infty,-1] \cup[0, b] \cup[c, \infty)) \\
& =\rho_{\alpha}\left(A, T^{\prime} T B \mid(-\infty,-1] \cup\left[0, b_{1}\right] \cup\left[c_{1}, \infty\right)\right) \\
& =\rho_{\alpha}\left(A, T^{\prime \prime} T^{\prime} T B \mid(-\infty,-1] \cup\left[0, b_{2}\right] \cup\left[c_{2}, \infty\right)\right) \\
& =\cdots \\
& =\rho_{\alpha}\left(A, T^{(n)} \cdots T^{\prime \prime} T^{\prime} T B \mid(-\infty,-1] \cup\left[0, b_{n}\right] \cup\left[c_{n}, \infty\right)\right) \\
& =\cdots
\end{aligned}
$$

From the fact that

$$
\rho_{\alpha}^{2}(A, B \mid e)=1-\sigma^{2}(A \mid e \cup\{B\}) \sigma^{-2}(A \mid e) \quad \text { for } A, B \notin e,
$$

the $n+2$ nd member of the above equalities is dominated by

$$
\begin{gathered}
\left(1-\frac{\sigma^{2}\left(A \mid(-\infty,-1] \cup\left[0, b_{n}\right] \cup\left[c_{n}, \infty\right) \cup\left\{B_{n}\right\}\right)}{\sigma^{2}\left(A \mid(-\infty,-1] \cup\left[0, b_{n}\right] \cup\left[c_{n}, \infty\right)\right)}\right)^{1 / 2} \\
\leqq\left(1-\frac{\sigma^{2}(A \mid(-\infty,-1] \cup[0, \infty))}{\sigma^{2}\left(A \mid(-\infty,-1] \cup\left[0, b_{n}\right]\right)}\right)^{1 / 2},
\end{gathered}
$$

where $B_{n}=T^{(n)} \cdots T^{\prime \prime} T^{\prime} T B$. Letting $b_{n} \rightarrow \infty$, we prove

$$
\sigma^{2}\left(A \mid(-\infty,-1] \cup\left[0, b_{n}\right]\right) \longrightarrow \sigma^{2}(A \mid(-\infty,-1] \cup[0, \infty)) \text {. }
$$

Consequently, we obtain $\rho_{\alpha}(A, B \mid(-\infty,-1] \cup[0, a])=0$ for any $A \in(-1,0)$ and any $B \in(a, \infty)$, and this fact contradicts the anti-Markov property of $\boldsymbol{X}_{\alpha}$.

(Q.E.D.)

\section{REFERENCES}

[1] H. Dym and H. P. McKean, Jr.: Application of de Branges spaces of integral functions to the prediction of stationary Gaussian processes, Ill. J. Math. 14 (1970), 299-343.

[2] M. I. Fortus: Formulas for extrapolation of random fields, Theory Prob. Appl. 7 (1962), 101-108. (English translation)

[ 3 ] R. Gangolli: Positive definite kernels on homogeneous spaces and certain stochastic processes related to Lévy's Brownian motion of several parameters, Ann. Inst. Henri Poincaré section B vol. III n. 2 (1967), 121-225.

[4] I. Kubo: Some topics on random fields, Seminar on Probability vol. 26 (1967). (In Japanese)

[ 5 ] P. Lévy: Processus stochastiques et mouvement Brownien, Gauthier-Villars, Paris (1965).

[6] H. P. McKean, Jr.: Brownian motion with a several-dimensional time, Theory Prob. Appl. 8 (1963), 335-354. 
[ 7 ] G. M. Molchan: Characterization of Gaussian fields with Markov property, Dokl. Akad. Nauk. SSSR 197 (1971), 784-787.

[8] G. M. Molchan and Ju. I. Golosov: Gaussian stationary processes with asymptotic power spectrum, Dokl. Akad. Nauk. SSSR 184 (1969), 546-549.

[9] A. M. Yaglom: Correlation theory of processes with random stationary n-th increments, Amer. Math. Soc. Transl. Ser 2 Vol. 8 (1958), 87-141.

[10] A. M. Yaglom: Some classes of random fields in n-dimensional space related to stationary random processes, Theory Prob. Appl. 2 (1957), 273-320. (English translation)

Aichi University of Education 